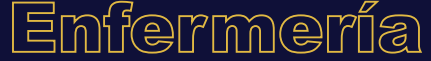

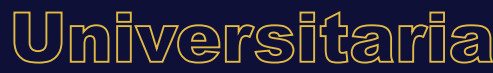

\section{La simulación clínica en la adquisición de conocimientos en estudiantes de la Licenciatura de Enfermería}

\section{Clinical simulation in acquisition of knowledge among undergraduate nursing students}
A simulação clínica na aquisição de conhecimentos em estudantes da Licenciatura de Enfermagem

\section{Amaro-López ${ }^{\mathrm{a}^{*}}$, P.L. Hernández-González ${ }^{\mathrm{b}}$,} A. Hernández-Blas ${ }^{c}$, L.I. Hernández-Arzola ${ }^{\mathrm{d}}$

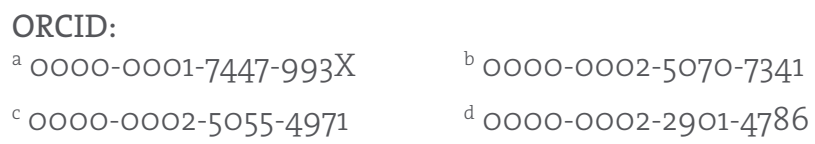
diantes de enfermería experimentar la representación de un hecho real, para practicar, aprender y adquirir los conocimientos necesarios para el desempeño de su profesión. En Oaxaca, la Universidad de la Sierra Sur (UNSIS), tiene una clínica robotizada, que apoya a los estudiantes en el desarrollo de su práctica clínica, pero aún no cuenta con una metodología específica para llevar a cabo simulación clínica.

Objetivo: Comparar la adquisición de conocimientos en estudiantes de enfermería con el método tradicional y otro basado en simulación clínica. 
Metodología: Estudio de pre y postest para evaluar conocimiento del tema trabajo de parto en alumnos de $4^{\circ}$ semestre; se compararon dos metodologías, la tradicional utilizada en la universidad y otra basada en simulación clínica; la primera consistió en dar la clase teórica en aula y replicarla con un simulador; la segunda consistió en clase teórica, escenario de simulación clínica, caso clínico y debriefing.

Resultados: Hubo diferencia estadísticamente significativa ( $p<.001)$ entre pretest y postest en ambas metodologías, pero quienes recibieron la metodología basada en simulación clínica obtuvieron mayores puntajes ( $p=0.004)$.

Discusión: El uso de una metodología basada en simulación clínica permitió que los alumnos obtuvieran mayores conocimientos en el tema trabajo de parto, respecto a la metodología tradicional que se utiliza en la universidad. La UNSIS empieza a generar evidencia sobre el uso de la simulación clínica adaptada a su modelo educativo.

Conclusiones: Utilizar la simulación clínica logró que los estudiantes obtuvieran mayores conocimientos.

Palabras Clave: Simulación clínica; conocimiento; educación en enfermería; México.

\section{ABSTRACT}

Introduction: Clinical simulation is an educational tool which allows nursing students to experience representations of real events in order to practice, learn, and acquire the necessary knowledge to perform in their profession. In Oaxaca, Mexico, the Universidad de la Sierra Sur (UNSIS), has a robotic clinic which helps nursing students in their clinical learning; however, a specific clinical simulation methodology needs to be developed.

Objective: To contrast the acquisition of knowledge among nursing students as a result of using the traditional method in comparison to using a method based on clinical simulation. Methodology: This is pre and post-test study to assess the knowledge development related to labor among nursing students from the 4 th semester. Two teaching methodologies were compared: the traditional one, which included classroom classes and a replication using a simulator, and the clinical simulation based one, which included classroom classes, a clinical simulation scenario, a clinical case, and a debriefing session.

Results: Statistically significant differences ( $\mathrm{p}<.001$ ) were found between the pre and post test knowledge status with both teaching methodologies; but the improvement of those who learned through the clinical simulation based methodology was higher $(\mathrm{p}=0.004)$.

Discussion: The use of the methodology based on clinical simulation allowed the students to acquire more knowledge on labor among in comparison to the use of the traditional methodology. The UNSIS is now collecting evidence on the use of clinical simulation in order to adapt this methodology to its educational model.

Conclusion: The use of clinical simulation resulted in the acquisition of more knowledge by the nursing students.

Keywords: Clinical simulation; knowledge; education, nursing; Mexico.

\section{RESUMO}

Introdução: A simulação clínica é uma ferramenta educativa que permite aos estudantes de enfermagem experimentar a representação de um fato real, para praticar, aprender e adquirir os conhecimentos necessários para o desempenho de sua profissão. Em Oaxaca, a Universidad de la Sierra Sur (UNSIS), tem uma clínica robotizada, que apoia aos estudantes 
no desenvolvimento de sua prática clínica, mas ainda não conta com uma metodologia específica para levar a cabo simulação clínica.

Objetivo: Comparar a aquisição de conhecimentos em estudantes de enfermagem com o método tradicional e outro baseado na simulação clínica.

Metodologia: Estudo de pre e postest para avaliar conhecimento do tema trabalho de parto nos alunos de $4^{\circ}$ semestre; compararam-se duas metodologias, a tradicional utilizada na universidade e outra baseada em simulação clínica; a primeira consistiu em dar a aula teórica na sala e replicá-la com um simulador; a segunda consistiu na aula teórica, cenário de simulação clínica, caso clínico e debriefing.

Resultados: Houve diferença estatisticamente significativa ( $p<.001)$ entre pretest e postest em ambas as metodologias, mas aqueles que receberam a metodologia baseada em simulação clínica obtiveram maiores pontuações ( $p=0.004$ ).

Discussão: O uso de uma metodologia baseada em simulação clínica permitiu que os alunos obtivessem maiores conhecimentos no tema trabalho de parto, ao respeito da metodologia tradicional que se utiliza na universidade. A UNSIS começa a gerar evidência sobre o uso da simulação clínica adaptada a seu modelo educativo.

Conclusões: Utilizar a simulação clínica conseguiu que os estudantes obtivessem maiores conhecimentos.

Palavras Chave: Simulação clínica; conhecimento; educação em enfermagem; México.

\section{INTRODUCCIÓN}

La educación en el área de la salud es un proceso cambiante y complejo, donde se exige la incorporación de nuevos métodos de enseñanza y aprendizaje $e^{1}$. La simulación clínica como método educativo ha estado siempre presente en la formación de profesionales de enfermería ${ }^{2}$. A principios del siglo $\mathrm{XX}$, los estudiantes de enfermería tenían las denominadas salas de arte, sitios destinados para realizar prácticas y desarrollar algunos procedimientos con el fin de mejorar la atención del paciente 3 . Gracias a la constante evolución de la simulación aplicada a las ciencias de la salud, se fueron creando simuladores cada vez más sofisticados; por ejemplo, en 1911 se creó la Señora Chese, para 1960 aparecieron los simuladores Sime One, en el 2000 se crearon el Sim MAN y NOELLE ${ }^{\circledR}$, para el 2010 se generaron los simuladores Haptic Simulators, con características que se acercan más a la realidad4-6.

La simulación clínica ha resultado ser una herramienta educativa con una metodología innovadora basada en un modelo de enseñanza constructivista ${ }^{7,8}$, donde el conocimiento no se descubre, se construye. En consecuencia, el estudiante participa activamente en su proceso de aprendizaje al realizar de manera segura y controlada una práctica muy parecida a la realidad ${ }^{9}$, que contribuye a la adquisición de conocimientos, habilidades y competencias ${ }^{10}$.

A su vez el modelo de Miller evalúa las competencias organizándolas en una pirámide de cuatro niveles por orden de complejidad ${ }^{7,11}$; en la base están aquellos que corresponden a conocimientos (el saber) y competencias (saber cómo); en los niveles superiores se encuentran mostrar cómo (demuestra cómo lo haría) y el hacer (se observa el desempeño en situaciones reales); es decir, los conocimientos se entenderán como la base fundamental, para lograr una competencia de mayor complejidad, como es el desempeño y la acción en la práctica real.

Martínez y Matus $^{12}$ mencionan que la simulación clínica ha acompañado a la enseñanza en enfermería desde hace tiempo, pero la incorporación de la tecnología conduce a la integración de 
conocimientos, habilidades y destrezas, previo a estar en contacto con el paciente real, lo que permite una mayor autonomía y dirección en su profesión ${ }^{13,14}$. Por su parte, Piña y Amador ${ }^{15}$, afirman que la simulación clínica en enfermería es una herramienta que apoya a la práctica clínica del estudiante y está debe contemplar elementos indispensables para su uso, por ejemplo: presentación conceptual, abordaje de contenidos, objetivos, planeación, creación de escenarios y debriefing.

Diferentes autores describen las metodologías que emplearon en sus estudios con la finalidad de demostrar los beneficios de la simulación clínica en la enseñanza y aprendizaje de estudiantes de enfermería, entre ellos se encuentran:

$\operatorname{Vargas}^{16}$, que empleó talleres formativos organizados en escenarios donde los estudiantes de diferentes semestres, según el nivel de conocimientos, desarrollaron con su respectivo docente la práctica simulada y contaron con el apoyo de una enfermera que brindó asesorías tanto al docente como a los alumnos.

Martínez y Matus ${ }^{12}$ utilizaron casos clínicos simulados, en donde los estudiantes realizaban el rol de enfermería a cargo del paciente y debían tomar las decisiones para realizar las intervenciones más adecuadas.

Juguera et $a 1^{17}$ emplearon escenarios clínicos según la práctica que los alumnos cursaban en ese momento, dispusieron de salas de simulación y el área del debriefing. Los estudiantes atendieron una serie de intervenciones extraídas de la Nursing Interventions Classification (NIC) a través de la implementación de un caso clínico, esto se registró en video que se utilizó para llevar a cabo el debriefing inmediatamente después de la simulación, el instructor fue un profesor de enfermería, que controló mediante un sistema informático y software específico el simulador y el desarrollo del escenario desde la sala control.

Velasco ${ }^{18}$ utilizó en su estudio todas las fases didácticas; la sesión informativa previa, introducción, reunión sobre el manejo del simulador, teoría, reunión o discusión sobre el caso, escenario, debriefing y conclusión.

Niño et $\mathrm{al}^{13}$ emplearon una metodología que tuvo como propósito brindar herramientas pedagógicas en simulación clínica para el fortalecimiento de las habilidades técnicas y competencias de los estudiantes de enfermería. Esta consistió en realizar un diagnóstico en docentes y estudiantes para determinar los temas a tratar en los talleres de simulación. Se elaboró una programación semanal de actividades, se organizaron los espacios físicos y se adecuaron los simuladores. Con este estudio se evidenció la satisfacción de los estudiantes respecto al uso de la simulación clínica durante su enseñanza, pues estos expresaron la necesidad de realizar talleres adicionales sobre procedimientos específicos para fortalecer la práctica de enfermería; además se generaron guías de simulación clínica.

Gracias a los beneficios alcanzados, la Organización Mundial de la Salud ha apoyado para que se extienda el uso de la simulación clínica en toda América Latina, con el objetivo de mejorar la calidad de la enseñanza y el aprendizaje de los estudiantes de enfermería ${ }^{19}$.

En Oaxaca, la Universidad de la Sierra Sur (UNSIS) cuenta con una clínica robotizada donde los profesores utilizan simuladores de baja y alta complejidad para desarrollar las diferentes técnicas y procedimientos propios de enfermería, sin embargo; hoy en día no existe aún una metodología para implementar simulación clínica en esta institución.

La presente investigación permite generar evidencia sobre el uso de la simulación clínica en la adquisición de conocimientos en estudiantes de enfermería al implementar la metodología de Dura Ros4, la cual se adapta mejor a los objetivos de nuestro estudio por emplear pretest y postest al proceso de simulación, la comparación entre grupos y el desarrollo del debriefing en fases. 
La UNSIS tiene un modelo de tiempo completo ${ }^{20}$, los alumnos asisten a la escuela en la mañana y en la tarde, en sus horarios se incluyen las clases, horas de biblioteca, de cómputo, inglés, club y tutorías, por lo que prácticamente no disponen de tiempo extra. Los grupos de la Licenciatura en Enfermería son numerosos con un aproximado de 30 a 35 alumnos, el tiempo estimado para la clase y la práctica es de una hora, junto con el contenido teórico se aborda el contenido práctico; es decir, cuando se imparte la teoría del tema trabajo de parto durante la semana se tendrá que hacer la práctica en la clínica robotizada, donde el profesor da la clase con el simulador y los estudiantes son observadores de la técnica y procedimiento. Debido a esto, resulta complicado llevar a cabo los procedimientos de manera individual y verificar si el alumno realmente tiene los conocimientos sobre lo que realiza.

Por lo anterior, el objetivo del presente estudio fue comparar la metodología que tradicionalmente se utiliza en la UNSIS, respecto a una metodología basada en simulación clínica propuesta por Dura Ros4, y así poder establecer con cuál metodología el alumno adquiere mayores conocimientos. Cabe señalar que está tuvo que ser adaptada al modelo de la universidad debido a la complejidad de los tiempos que se estipulan para cada práctica y la cantidad de alumnos por grupo.

\section{MÉTODOS}

Es un estudio de pretest y postest, en el que participaron 119 alumnos de $4^{\circ}$ semestre que cursaban la materia Enfermería Materno Infantil. El escenario fueron las aulas y la clínica robotizada de la UNSIS entre marzo y julio de 2017.

\section{Diseño y aplicación del instrumento}

Se realizó una guía para el desarrollo del escenario de simulación clínica de acuerdo con lo propuesto por Dura y Velasco ${ }^{4,18}$ se incluyeron elementos como: nombre del escenario, autores, participantes, lugar, fecha, cronograma, objetivos, descripción del escenario, personas que participarían en el escenario, preparación del escenario, simulador y los acontecimientos a realizar, actitud de los participantes, el debriefing (sesión posterior para la discusión de la práctica realizada), que consistió de tres apartados: fase de descripción, análisis y aplicación, así como la finalización de la simulación clínica.

El cuestionario de conocimientos y el caso clínico se realizaron apegándose al contenido del programa de la materia Enfermería Materno Infantil, impartida a los estudiantes en el $4^{\circ}$. semestre, específicamente se abordó el tema atención al trabajo de parto, éstos fueron revisados por profesores titulares de la materia. El cuestionario final quedó constituido por dos secciones, en la primera se recabó información sociodemográfica: nombre, sexo, fecha de nacimiento, años cumplidos, si estaban recursando la materia (ya que esto es criterio de exclusión), región de procedencia y si hablaban una lengua indígena; la segunda sección estuvo conformada por 13 preguntas de opción múltiple que evaluaban el tema. El caso clínico consistió en una paciente en trabajo de parto sin complicaciones.

A los estudiantes se les presentó el objetivo del estudio y se les invitó a participar, quienes decidieron hacerlo firmaron una carta de consentimiento informado, posteriormente se les aplicó el cuestionario de conocimientos acerca del tema atención del trabajo de parto con la finalidad de hacer un diagnóstico de los conocimientos que tenían, el mismo profesor fue el encargado de impartir la clase teórica en el aula y en la clínica robotizada. De los cuatro grupos participantes, cada uno aproximadamente de 30 alumnos, se seleccionaron dos grupos en forma aleatoria que recibieron la metodología de la enseñanza tradicional, la cual consistió en que el profesor diera la clase teórica en el aula e impartiera la práctica con el simulador Victoria S2200 en la clínica robotizada, los alumnos 
sólo observaron y la actividad finalizó cuando el profesor terminó ambas clases. Los otros dos grupos recibieron la metodología basada en la simulación clínica descrita por Dura Ros4, que consistió al igual que en la enseñanza tradicional, en una clase teórica y la práctica en la clínica robotizada pero a diferencia de la desarrollada con la enseñanza tradicional, contó con la participación de estudiantes y se desarrolló de la siguiente manera: se llevó acabo la introducción al escenario de simulación, la representación de un caso clínico, se seleccionaron dos alumnos al azar esto debido al tiempo para realizar la práctica y la cantidad de estudiantes que había por grupo, se les explicó el caso para escenificar la atención del trabajo de parto con el simulador Victoria S2200 (simulador de alta fidelidad que se programó para dar repuestas como dolor, el grito en cada contracción y la acción de pujar, la sangre a la salida del producto, etc.), se les explicó que uno de ellos tomaría el rol de enfermera(o) que atendería el parto y el otro el de enfermera(o) asistencial, los alumnos restantes sólo observaron ya que se contaba con 30 minutos para realizar la práctica.

Una vez concluida la simulación se llevó a cabo el debriefing con una duración de 30 minutos, para ello se dividió al grupo en dos, la mitad de los estudiantes se quedó con el profesor a cargo y la otra mitad con el instructor, ambos siguieron el guion propuesto por Dura Ros4, con las fases de descripción, análisis y aplicación, con esto se finalizó la simulación clínica. Con la finalidad de evaluar el grado de retención, a los alumnos se les aplicó nuevamente un cuestionario de conocimientos tres semanas después.

Para el análisis estadístico las variables categóricas se describieron en porcentajes, las variables cuantitativas con media y desviación estándar. Las comparaciones entre los grupos se efectuaron mediante ANOVA y se consideró un nivel de significancia de $p=<0.05$.

La base de datos se realizó con el programa excel, y el procesamiento estadístico con el programa JMP versión 8.

\section{RESULTADOS}

Participaron 119 estudiantes, de ellos 11 fueron descartados por tener conocimientos previos al estar recursando la materia y 12 porque solo contestaron un cuestionario o no lo completaron adecuadamente, por ello la muestra final constó de 96 alumnos, de los cuales el 48\% $(n=46)$ recibieron el tema con el método tradicional y el $52 \%(n=50)$ con el método basado en simulación clínica. La distribución por sexo en la metodología tradicional fue de $83 \%$ mujeres y $17 \%$ hombres; en la metodología basada en simulación clínica correspondió a $80 \%$ mujeres y 20\% hombres. La edad promedio fue de

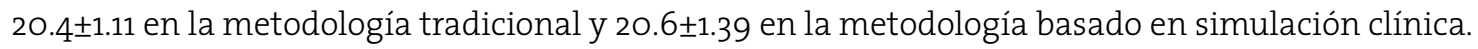

Existen alumnos de casi todas las regiones del estado; aunque la mayoría proviene de los Valles Centrales y de la Sierra Sur, región en la que se localiza la Universidad, solamente el 1\% de los estudiantes proviene de otro estado de la República.

De los alumnos que pertenecen a una etnia indígena, el 6.2\% habla chinanteco, el 12.5\% chatino, el 12.5\% mazateco, el 18.8\% mixe y el 50\% zapoteco.

La gráfica 1 nos muestra la diferencia de medias en el pretest entre quienes recibieron el tema con la metodología tradicional y quienes lo recibieron con la metodología basada en simulación clínica; como se observa, son prácticamente similares.

El postest se aplicó tres semanas después de realizar la simulación; la gráfica 2 nos muestra la diferencia de medias entre el pretest y el postest de quienes recibieron el tema con la metodología tradicional; se observa una diferencia estadísticamente significativa $(p<.001)$ entre los puntajes obtenidos en ambas evaluaciones. 


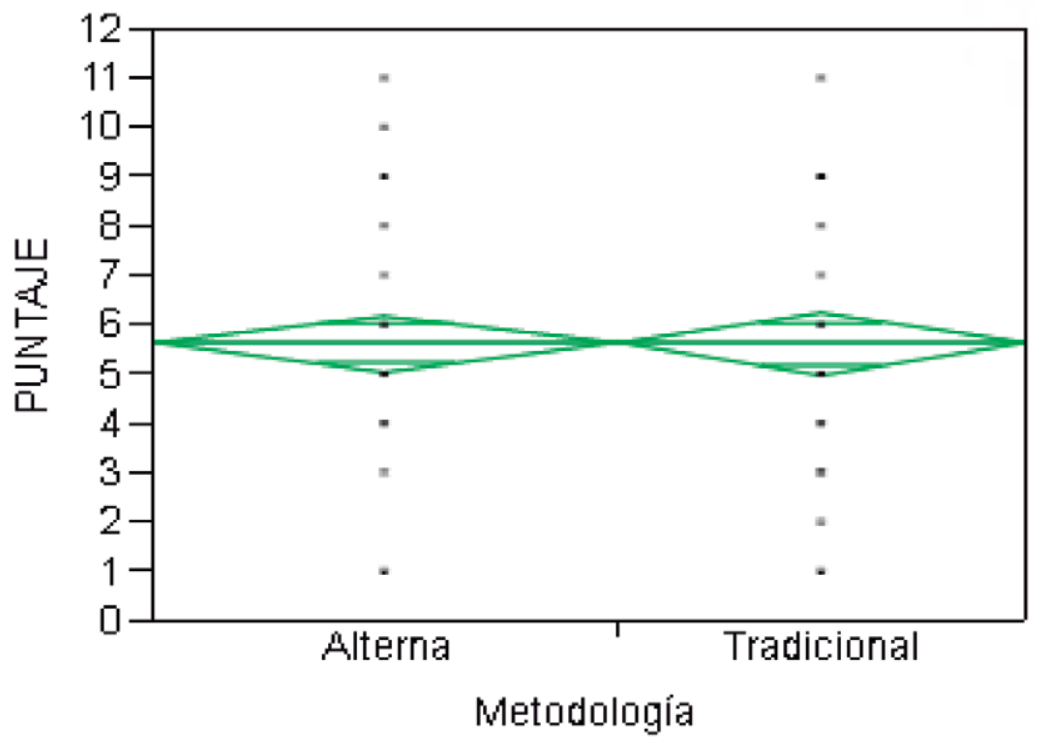

Gráfica 1. Diferencia de medias entre la metodología tradicional y la metodología alterna en el pretest

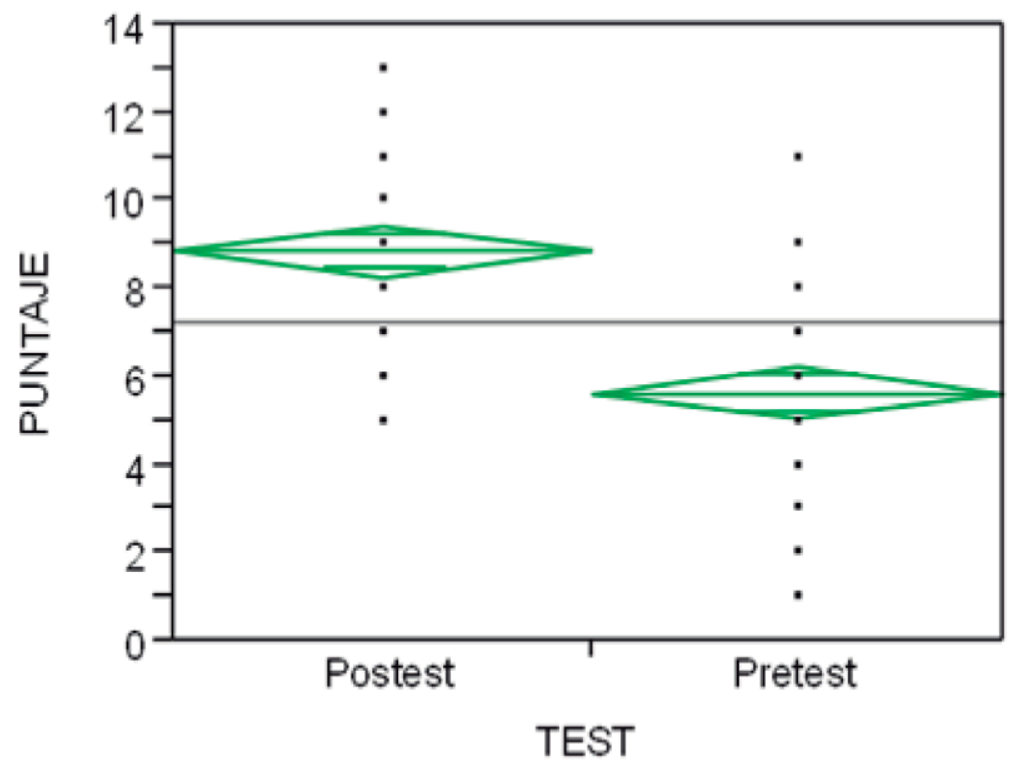

Gráfica 2. Diferencia de medias entre pretest y postest de los estudiantes que recibieron la metodología tradicional.

En la gráfica 3 se presenta la diferencia de medias entre ambas evaluaciones de quienes recibieron el tema con la metodología basada en simulación clínica; también hubo una diferencia estadísticamente significativa ( $p<.001)$.

En la gráfica 4 se aprecia la diferencia de medias en el postest entre ambas metodologías, en donde se observa que quienes recibieron el tema con la metodología basada en simulación clínica obtuvieron mejores puntajes; esta diferencia fue estadísticamente significativa ( $p=0.004)$.

Durante el debriefing, en la fase de descripción, a pesar de que solo dos alumnos fueron sometidos al escenario, los demás mostraron empatía, refirieron también haberse sentido nerviosos y estresados ante lo realizado por sus compañeros. En la fase de análisis detectaron cosas importantes 


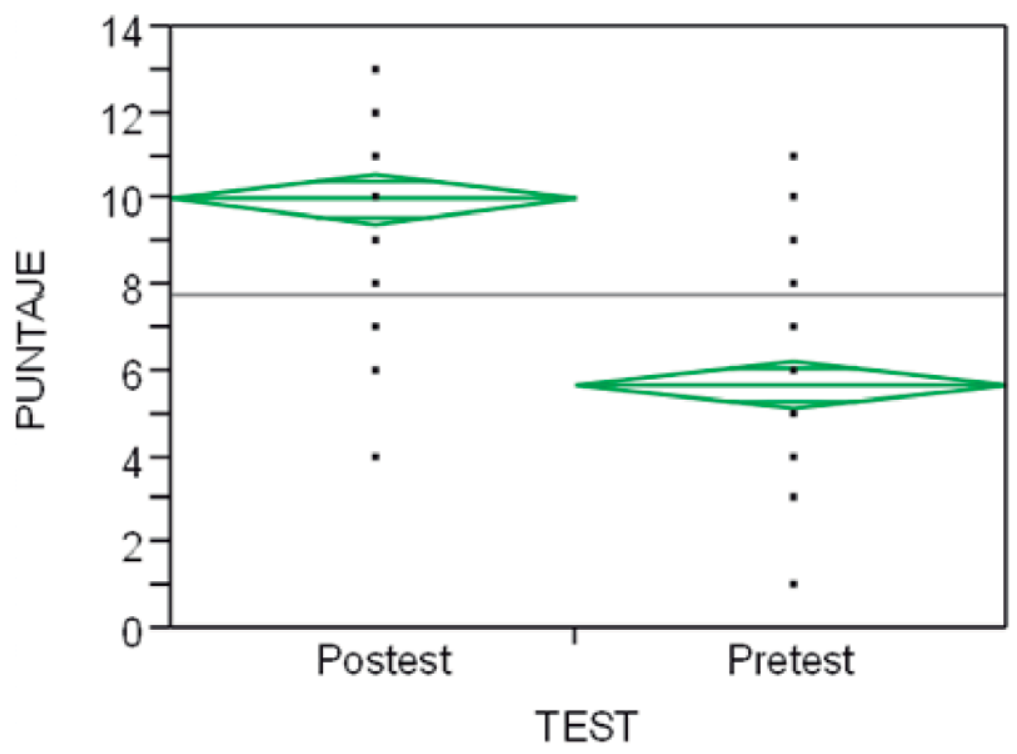

Gráfica 3. Diferencia de medias entre pretest y postest de los estudiantes que recibieron la metodología alterna

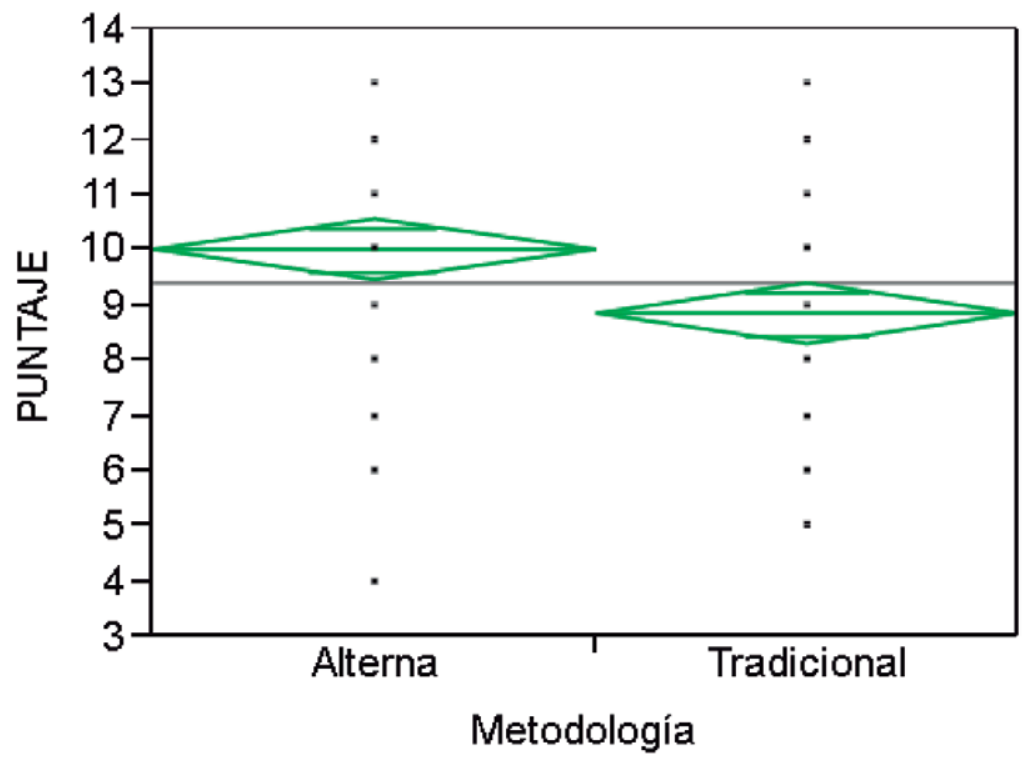

Gráfica 4. Diferencia de medias entre la metodología tradicional y la metodología alterna en el postest

como el trabajo en equipo es importante para la atención de los pacientes, y finalmente mencionaron que la simulación es ver qué es lo que se debe y no se debe hacer en la realidad con el paciente; los dos estudiantes que participaron directamente con el simulador refirieron sentirse ahora más seguros ante el procedimiento.

También se analizó si había una diferencia entre los estudiantes que hablaban otra lengua aparte del español y quienes no lo hacían, para saber si esta habilidad incidía de alguna forma en el aprendizaje, pero los puntajes fueron muy similares. 


\section{DISCUSIÓN}

El uso de la simulación clínica en el proceso de enseñanza aprendizaje permite que los alumnos adquieran conocimientos significativos ${ }^{21}$. Dentro de la literatura existen diferentes metodologías de enseñanza donde se emplea la simulación clínica, estas se adaptan al nivel de conocimientos, al personal con que se cuenta, al espacio y tiempo con que se disponga, entre otras circunstancias. En la UNSIS, los profesores hacen uso de los simuladores para enseñar a sus estudiantes los diversos procedimientos que se abordaran en sus asignaturas, pero todavía falta implementar metodologías que permitan hacer simulación clínica con todos los aspectos descritos por Dura4, lo que puede ser una fortaleza en la enseñanza y aprendizaje de la Universidad.

Otro aspecto importante a señalar es que hasta el momento no se había estudiado si los alumnos verdaderamente adquieren los conocimientos necesarios para desarrollar su práctica clínica, la propuesta que se plantea es que con el modelo de tiempo completo que maneja la universidad, se pueda empezar a implementar simulación clínica. En el presente trabajo se observó que el uso de la simulación clínica basada en el modelo de Dura permitió que el alumno tuviera mayores conocimientos y un mejor aprendizaje.

López et al $^{22}$ y Ruiz ${ }^{23}$ reportan, que al utilizar la simulación clínica los alumnos adquieren un mejor aprendizaje, incrementan su capacidad cognitiva y analítica, también adquieren habilidades técnicas de comunicación y razonamiento, ya que reconocen sus errores, reflexionan sobre los mismos y corrigen las fallas que se ocasionaron, para así desarrollar una práctica futura de calidad y competencia con el fin de posibilitar el trabajo en equipo de forma coordinada. A esto se añade lo referido por Martínez et al. ${ }^{12}$, quienes señalan a la simulación como una experiencia valiosa porque ha permitido capacitar a equipos multidisciplinarios para brindar una atención médica de calidad y más segura para el paciente. Juguera ${ }^{17}$ en su estudio menciona que el debriefing promovió actividades como pensamiento crítico, responsabilidades y capacidad para resolver problemas.

Durante el debriefing, los estudiantes mencionaron que al escenificar un caso clínico y posteriormente analizarlo, se sintieron más involucrados en el procedimiento del trabajo de parto, pudieron compartir abiertamente sus experiencias y opiniones al analizar los aciertos y errores, expresaron que esto les ayudará a mejorar en sus prácticas clínicas futuras, lo que concuerda con el estudio de Solórzano ${ }^{24}$, quién menciona que el debriefing hace que los estudiantes aprendan de los errores, les ayuda a corregir de manera significativa la información y su ejecución, lo que repercutirá en la mejora de la técnica. De igual forma, durante nuestro estudio se observó que el docente se involucró más con el alumno al promover un aprendizaje significativo, que hizo que los estudiantes mejoraran sus conocimientos, con ello se logró el objetivo primordial del estudio; y aunque dentro de los objetivos no se estableció la evaluación de competencias, se observó más apoyo, comprensión, empatía y mejor comunicación entre los estudiantes.

Así mismo, la simulación clínica ha permitido disminuir la brecha entre la teoría y la práctica. Por lo tanto, conviene enfatizar la importancia de generar escenarios clínicos lo más cercano a la realidad, que permitan la participación directa del estudiante y lo inviten a la reflexión para la adquisición de conocimientos, habilidades y competencias necesarias de acuerdo a cada procedimiento desarrollado, con la finalidad de desempeñarse en la práctica real lo mejor posible, con atención de calidad y seguridad para el paciente. La simulación clínica en la educación de enfermería, tiene muchas ventajas: adquisición de conocimientos, el desarrollo de autoconfianza, incentivo de trabajo en equipo, aumento de habilidades de pensamiento crítico, ambiente controlado y seguro, 
retroalimentación inmediata sobre lo realizado, experiencia de aprendizaje interactivo y articulación de la teoría con la práctica clínica²5.

Los avances en la simulación van de manera creciente, en muchos hospitales y universidades está siendo incorporada como una metodología para la validación de procedimientos, mejorar la atención del paciente con la disminución del error y la integración en los planes de estudios para los procesos de evaluación institucional.

\section{CONCLUSIONES}

En este estudio, los alumnos que recibieron el tema de trabajo de parto con la metodología de simulación clínica de Dura obtuvieron mejores puntajes en el cuestionario de conocimientos acerca del tema; en comparación con los alumnos que solo recibieron el tema con el apoyo del simulador.

Los resultados de esta investigación sugieren implementar la simulación clínica en las diferentes prácticas de las carreras afines al área de salud que trabajan directamente con el paciente, a fin de mejorar el aprendizaje del estudiante y la enseñanza docente, se recomienda la creación de pequeños grupos para tener un mejor control en el trabajo de simulación, talleres adicionales según la práctica que corresponda y que el alumno pueda practicar continuamente, la elaboración de guías de simulación que orienten tanto al profesor como al alumno y, adecuar espacios para llevar a cabo cada una de las fases didácticas de la simulación.

Finalmente, una de las limitaciones del estudio fue que solo se enfocó en evaluar conocimiento, faltó evaluar habilidades y competencias, por lo que se sugiere que en próximos trabajos se tomen en cuenta estos elementos.

\section{RESPONSABILIDADES ÉTICAS}

Protección de personas y animales. Los autores declaran que para esta investigación no se han realizado experimentos en seres humanos ni en animales.

El proyecto fue presentado y aprobado por el comité de ética de la UNSIS.

Confidencialidad. Los estudiantes que participaron en la presente investigación firmaron un consentimiento informado en el cual se les hizo saber que la información que proporcionarían se manejaría de manera confidencial. En este trabajo no aparecen datos de los alumnos.

Conflicto de intereses. Los autores declaran no tener conflicto de intereses.

Financiamiento. Ninguno.

Agradecimientos. A las autoridades de la institución educativa por la apertura de las instalaciones empleadas para llevar a cabo dicho estudio y al personal técnico de la clínica robotizada

\section{REFERENCIAS}

1. Barrios-Araya S, Masalán-Apip MP, Cook MP. Educación en Salud: en la búsqueda de metodologías innovadoras. Cienc. enferm. 2011;17 (1): 57-69. http://dx.doi.org/10.4067/So717-95532011000100007

2. Urra-Medina E, Sandoval-Barrientos S, Irribarren-Navarro F. El desafío y futuro de la simulación como estrategia de enseñanza en enfermería. Investigación educ. médica 2017; 6(22): 119-25. http://dx.doi.org/10.1016/j.riem.2017.01.147

3. Quirós-Mora S, Vargas-de Oliveira MA. Simulación Clínica: una estrategia que articula prácticas de enseñanza e investigación en Enfermería. Texto contexto enferm. 2014; 23(4): 817-18.

http://dx.doi.org/10.1590/0104-0707201400120oedt. 
4. Durá-Ros MJ. La simulación clínica como metodología de aprendizaje y adquisición de competencias en enfermería [Tesis de Doctorado]. Madrid: Universidad Complutense de Madrid; 2013.

http://bit.ly/335basI

5. De la Horra-Gutiérrez I, Beneit-Montesinos JV. La simulación clínica como herramienta de evaluación de competencias en la formación de enfermería. Reduca (Enfermería, Fisioterapia y Podología). 2010; 2(1): 549-80. http://bit.ly/329WK9y

6. Corvetto M, Bravo MP, Montaña R, Utili F, Escudero E, Boza C, et al. Simulación en educación médica: una sinopsis. Rev. méd. Chile. 2013; 141(1): 70-9. http://bit.ly/36lXJ9R

7. Enriquez, DS, Perretta, C. Enseñanza de la Pediatría con simuladores. IntraMed j. 2014; 3(2): 8 pantallas. https://bit.ly/2NxOZok

8. Díaz-Agea JL, Leal-Costa C, García-Méndez JA. Metodología de autoaprendizaje en entornos simulados (MAES@). Evidentia. 2014; 11(45): 6 pantallas. https://bit.ly/36m3Mv4

9. Ruda-Rodríguez NL. Simulación clínica en la mediación pedagógica y su relación con la práctica clínica. Revista Investigación en Salud Universidad de Boyacá. 2014; 1(2): 231-43. https://doi.org/10.24267/23897325.125

10. Martínez-Esparza AC, Estrada-Zaleta F, Gómez-Meraz Y, Rubio-Martínez R. ¿El entrenamiento con simuladores permite un mejor manejo de eventos adversos en anestesia? Reporte de seis casos. An Med. 2016; 61 (1): 53-7. https://bit.ly/2PBKNXw

11. Puga-Tejada MA, Torres-Herrera CI. Perspectiva andragógica de la simulación clínica. Ciencia UNEMI. 2014; 7(12): 37-46. https://bit.ly/2NrNJDn

12. Martínez F, Matus R. Desarrollo de habilidades con simulación clínica de alta fidelidad. Perspectiva de los estudiantes de enfermería. Enferm. univ. 2015;12 (2): 93-8 http://dx.doi.org/10.1016/j.reu.2015.04.003

13. Niño-Herrera CA, Vargas-Molina NG, Barragán-Becerra JA. Fortalecimiento de la simulación clínica como herramienta pedagógica en Enfermería: Experiencia de Internado. Rev Cuid. 2015; 6(1): 970-5. http://dx.doi.org/10.15649/cuidarte.v6il.161

14. Rodríguez-Díez MC, Díez-Goñi N, Beunza-Nuin JJ, Auba-Guedea M, Olartecoechea-Linaje B, RuizZambrana A, et al. Confianza de los estudiantes de medicina en el aprendizaje de la exploración obstétrica con simuladores. An Sist Sanit Navar. 2013; 36(2): 275-80. http://dx.doi.org/10.4321/S1137-66272013000200010

15. Piña I, Amador R. La Enseñanza de la Enfermería con simuladores, consideraciones teórico pedagógicas para perfilar un modelo didáctico. Enferm. univ. 2015; 12(3): 152-9. http://dx.doi.org/10.1016/j.reu.2015.04.007

16. Vargas-Toloza ER. La simulación clínica, una experiencia para contar. Rev Cienc Cuidad. 2006; 3 (3), 112-8. http://bit.ly/32CVTOF

17. Juguera-Rodríguez L, Díaz-Agea JL, Pérez-Lapuente ML, Leal-Costa C, Rojo-Rojo A, Echevarría-Pérez P. La simulación clínica como herramienta Pedagógica. Percepción de los alumnos de grado en enfermería en la UCAM (Universidad Católica San Antonio de Murcia). Enferm Global. 2014; 13(1): 175-90. https://doi.org/10.6018/eglobal.13.1.157791

18. Velasco-Martín A. Simulación Clínica y Enfermería, creando un ambiente de simulación. [Trabajo de Grado]. Universidad de Cantabria; 2013. https://bit.ly/320utC1

19. Serna-Ojeda JC, Borunda-Nava D, Domínguez-Cherit G. La simulación en medicina. La situación en México. Cir Cir. 2012. 80(3); 301-5. https://bit.ly/2NtdLWL

20. Seara-Vazquez M. Un nuevo Modelo de Universidad. Universidades para el desarrollo. $2^{\mathrm{a}}$ ed. Oaxaca: Universidad Tecnológica de la Mixteca; 2010. p.178 
21. Alfonso-Mantilla JI,Martínez-Santa J.Modelos de simulación clínica para la enseñanza de habilidades clínicas en ciencias de la salud. Rev Mov Cient. 2015; 9(2): 70-9. http://bit.ly/2CFbnah

22. López-Sánchez M, Ramos-López L, Pato-López O, López-Álvarez S. La simulación clínica como herramienta de aprendizaje. Cir May Amb. 2013; 18 (1), 25-9. https://bit.ly/360HN6M

23. Ruiz-Dávila E. La enseñanza clínica en enfermería. Rev Mex Enf. 2015; 3 (1): 6-10. https://bit.ly/2N2kfNe

24. Solórzano-Torres FE, Perafán-Javela KT, Guerrero-Solorzano JA. Aplicación de checklist y debriefing en la técnica de aprendizaje de intubación endotraqueal neonatal. IntraMed j. 2016; 5 (2): 1-8. https://bit.ly/2N61TuE

25. Del Campo-Cazallas C, Fernández-Ayuso D, Galán-Lominchar M, De la Torre- Montero JC. Entornos de simulación como complemento para la evaluación de competencias de las Prácticas Tuteladas del Grado en Enfermería. Nure Inv. 2016; 13(81): 3-15. https://bit.ly/34iu2ED 